\title{
Rat Liver 10-formyltetrahydrofolate Dehydrogenase, Carbamoyl Phosphate Synthetase 1 and Betaine Homocysteine S-methytransferase were Co-purified on Kunitz-type Soybean Trypsin Inhibitor-coupled Sepharose CL-4B
}

\author{
Hyun-Sic Kim ${ }^{1, \#}$, Ji-man Kim ${ }^{2, \#}$, Kyung-Baeg Roh ${ }^{1}$, Hyeon-Hwa Lee ${ }^{1}$, Su-Jin Kim", \\ Young Hee Shin ${ }^{2}$ and Bok Luel Lee ${ }^{1, *}$ \\ ${ }^{1}$ College of Pharmacy, Pusan National University, Jangjeon Dong, Kumjeong Ku, Busan 609-735, Korea \\ ${ }^{2}$ College of Pharmacy, Kyung-Sung University, Daeyoun Dong, Nam-Ku, Busan 609-736, Korea
}

Received 9 April 2007, Accepted 17 April 2007

\begin{abstract}
An Asp/His catalytic site of 10-formyltetrahydrofolate dehydrogenase (FDH) was suggested to have a similar catalytic topology with the Asp/His catalytic site of serine proteases. Many studies supported the hypothesis that serine protease inhibitors can bind and modulate the activity of serine proteases by binding to the catalytic site of serine proteases. To explore the possibility that soybean trypsin inhibitor (SBTI) can recognize catalytic sites of FDH and can make a stable complex, we carried out an SBTI-affinity column by using rat liver homogenate. Surprisingly, the Rat FDH molecule with two typical liver proteins, carbamoyl-phosphate synthetase 1 (CPS1) and betaine homocysteine S-methyltransferase (BHMT) were co-purified to homogeneity on SBTI-coupled Sepharose and Sephacryl S-200 followed by Superdex 200 FPLC columns. These three liver-specific proteins make a protein complex with $300 \mathrm{kDa}$ molecular mass on the gel-filtration column chromatography in vitro. Immuno-precipitation experiments by using anti-FDH and anti-SBTI antibodies also supported the fact that FDH binds to SBTI in vitro and in vivo. These results demonstrate that the catalytic site of rat FDH has a similar structure with those of serine proteases. Also, the SBTI-affinity column will be useful for the purification of rat liver proteins such as FDH, CPS1 and BHMT.
\end{abstract}

\footnotetext{
Abbreviations: FDH, 10-formyltetrahydrofolate dehydrogenase; SBTI, soybean trypsin inhibitor; CPS1, carbamoyl-phosphate synthetase 1; BHMT, betaine homocysteine S-methyltransferase
}

"The first two authors made equal contributions to this work.

*To whom correspondence should be addressed.

Tel: 82-51-510-2809; Fax: 82-51-513-6754

E-mail: brlee@pusan.ac.kr
Keywords: Betaine homocysteine S-methytransferase, Carbamoyl phosphate synthetase 1, 10-formyltetrahydrofolate dehydrogenase, Serine protease, Serine protease inhibitor

\section{Introduction}

10-formyltetrahydofolate dehydrogenase (FDH) converts 10 formyltetrahydrofolate (10-formyl-THF) to tetrahydrofolate (THF) in a nicotinic acid dinucleotide phosphate $\left(\mathrm{NADP}^{+}\right)$dependent dehydrogenase reaction or an $\mathrm{NADP}^{+}$-independent hydrolase reaction (Bailey and Gregory, 1999). The hydrolase reaction is known to occur in a 310-amino-terminal domain of FDH, whereas the dehydrogenase reaction requires the fulllength enzyme (Cook et al., 1991). The amino-terminal domain of FDH shares some sequence identity with several other enzymes utilizing 10-formyl-THF, such as glycinamide ribonucleotide formyltransferase (GART) and 10-formyltetrahydrofolate- L-methionyl- tRNA formyltransferase (FMT) (Krupenko et al., 1997). Interestingly, as shown in Fig. 1, these enzymes have two strictly conserved amino acid residues, His and Asp in the putative catalytic center (Cook et al., 1991). The fact that the conserved Asp and His are essential amino acid residues for several 10-formyl-THFutilizing enzymes, implies that these enzymes might have similar catalytic mechanisms (Inglese et al., 1990; Cook et al., 1991).

Serine proteases are one of the four major classes of proteolytic enzymes and have been studied in great detail in several physiological systems including digestion, coagulation, immune and endocrine function and fertilization (Perona and Craik, 1995; Iwanaga and Lee, 2005). The crystal structures of several serine proteases also revealed the importance of the three residues, Asp, His and Ser, which form the "catalytic triad" of serine proteases (Fig. 1), suggesting that 10-formyl- 


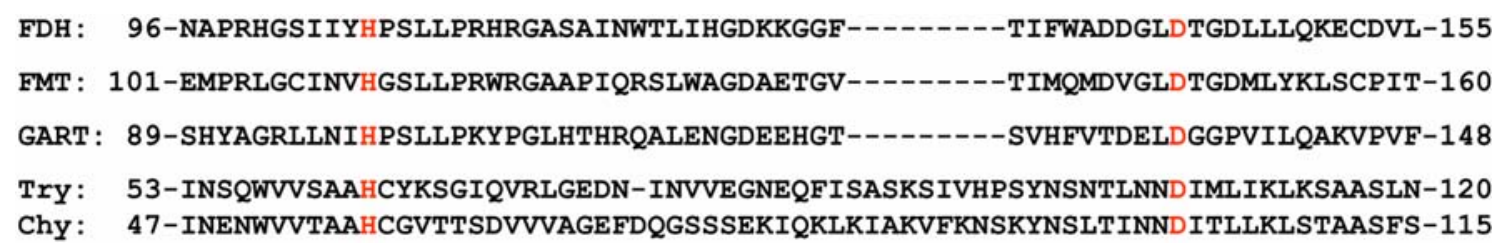

Fig. 1. Amino acid sequences similarities between rat FDH, E. coli FMT, E. coli GART, bovine trypsin (Try) and bovine chymotrypsin (Chy). Gaps (-) are inserted to maximize the sequence alignment. The conserved Asp and His residues were highlighted by red color.

THF-utilizing enzymes and serine proteases may have common structural elements for their enzyme catalysis (Murthy et al., 1999; Chumanevich et al., 2004). However, this hypothesis was not tested and not proved. Serine protease inhibitor (SPI) is serine protease-specific and binds tightly to the activated enzyme, blocking its protease activity (Prezelj et al., 2007). The SPIs can be divided into two functional classes on the basis of their interaction with the target serine proteases: irreversible 'trapping' reactions and reversible tight-binding reactions (Christeller, 2005). The former are specific to endopeptidases because their action depends on the cleavage of an internal peptide bond in the inhibitor that triggers a conformational change which traps the proteinase. The reversible tight-binding inhibitors form high-affinity but reversible interactions at the active site of the target enzyme, and are exemplified by serine and aspartyl protease inhibitors (Rawlings et al., 2004).

One of the well-known reversible SPIs is soybean trypsin inhibitor (SBTI) that contains the domain of a Kunitz trypsin inhibitor or a Bowman-Birk inhibitor. Many studies demonstrated that both inhibitors could inhibit the activity of several different serine proteases (Odani et al., 1979), indicating that SPIs can bind to several different serine enzymes and make the serine protease-inhibitor complex in vitro. These data allow us to hypothesize that FDH of rat liver can be obtained by using an SBTI-affinity column, because FDH may have a similar Asp/His pair as the active sites of a number of serine proteases. Here, we show for the first time that FDH binds to SBTI with another two rat liver proteins, carbamoylphosphate synthetase 1 (CPS1) and betaine homocysteine Smethyltransferase (BHMT) and that these three proteins subsequently make a complex in vitro and in vivo.

\section{Materials and Methods}

Animals. Male rats (200-300 g) were obtained from Harlan Sprague-Dawley and were maintained on a standard laboratory chow ad libitum. Animals were anesthetized with sodium pentobarbital $(50 \mathrm{mg} / \mathrm{kg}$ of body weight) before removal of the livers.

Preparation of rat liver homogenate. Rat livers were minced with a razor blade in homogenization buffer $(0.25 \mathrm{M}$ sucrose, $50 \mathrm{mM}$ Tris/HCl, pH 7.6, $14 \mathrm{mM}$ 2-mercaptoethanol and $2 \mathrm{mM}$ dithiothreitol containing $1 \mu \mathrm{g} / \mathrm{ml}$ each of antipain and leupeptin, $25 \mu \mathrm{M}$ amidinophenylmethanesulfonyl fluoride, and $10 \mu \mathrm{g} / \mathrm{ml}$ aprotinin). The minced livers were then homogenized four times (2-3 min/one time) by Polytron homogenizer (Brinkman) on an ice bath. The homogenates $(7.6 \mathrm{~g}$ protein $/ 190 \mathrm{ml})$ were centrifuged at $40,000 \times g$ for $2 \mathrm{~h}$ at $4^{\circ} \mathrm{C}$. The supernatant was then ultra-centrifuged (Beckman, $450,000 \times g$ for $2 \mathrm{~h}$ at $4^{\circ} \mathrm{C}$ ), and this solution was loaded onto SBTI-immobilized Sepharose CL-4B column for the purification of SBTI-binding proteins.

Preparation of SBTI-coupled Sepharose CL-4B resin. SBTI (Kunitz type, Sigma) was cross-linked to CNBr-Sepharose beads as described previously (Lee et al., 2004). Briefly, CNBr-Sepharose beads (Amersham Pharmacia Biotech) were swollen and washed in $1 \mathrm{mM} \mathrm{HCl}$. Beads $(1 \mathrm{~g})$ were transferred to coupling buffer (100 $\mathrm{mM} \mathrm{NaHCO}_{3}, \mathrm{pH} 8.3$, and $500 \mathrm{mM} \mathrm{NaCl}$ ) and added to $5 \mathrm{mg}$ of SBTI in coupling buffer. Coupling was carried out at room temperature for $1 \mathrm{~h}$. Residual active groups were blocked with $1 \mathrm{M}$ Tris- $\mathrm{HCl}, \mathrm{pH} \mathrm{8.0,} \mathrm{for} 2 \mathrm{~h}$ at room temperature. Beads were then washed successively and extensively four times in coupling buffer and acid wash buffer $(0.1 \mathrm{M}$ sodium acetate, $\mathrm{pH} 4.0$, and $500 \mathrm{mM}$ $\mathrm{NaCl}$ ).

Purification and characterization of SBTI-binding proteins from rat liver homogenate. To purify proteins that can recognize SBTI in the rat liver homogenate, the prepared homogenate solution was applied to an SBTI-immobilized Sepharose CL-4B column $(3.0 \times 15 \mathrm{~cm})$ equilibrated with $25 \mathrm{mM}$ Tris- $\mathrm{HCl}$, pH 7.6 (buffer A) at $0.5 \mathrm{ml} / \mathrm{min}$. After washing the column with buffer A until no absorbance at $280 \mathrm{~nm}$ was evident, bound proteins were eluted with a 0-300 $\mathrm{mM} \mathrm{NaCl}$ linear gradient. Fractions containing the $90 \mathrm{kDa}$ rat $\mathrm{FDH}$ protein were pooled, concentrated by ultrafiltration, and were then loaded onto a Sephacryl S-200 gel filtration column $(2.0 \times 130 \mathrm{~cm})$ equilibrated with buffer A containing $150 \mathrm{mM} \mathrm{NaCl}, \mathrm{pH} 7.6$ (buffer B) and washed with the same buffer. Fractions containing FDH were concentrated on a Centricon concentration filter (Amicon, Inc.) to a final volume of $0.5 \mathrm{ml}$. As a final purification step, a Superdex 200 FPLC gel filtration column $(0.8 \times 100 \mathrm{~cm})$ equilibrated with buffer B was used. The fractions containing SBTI-binding proteins were pooled and were analyzed by SDS-PAGE under reducing and nonreducing conditions. To determine partial amino acid sequences of the purified three proteins with molecular mass of $140 \mathrm{kDa}, 90 \mathrm{kDa}$ and $43 \mathrm{kDa}$, each band was cut out from the gel and then extracted by electro-elution with $250 \mathrm{~mA}$ for $3 \mathrm{~h}$ at $4^{\circ} \mathrm{C}$ according to the manufacturer's instructions. Finally, the purity of the electro-eluted three proteins was checked by SDS-PAGE under reducing conditions.

To determine the partial amino acid sequences of the purified 140 
$\mathrm{kDa}, 90 \mathrm{kDa}$ and $43 \mathrm{kDa}$ proteins, the purified proteins $(25 \mu \mathrm{g}$ each) were reduced, alkylated, and digested with $2 \mu \mathrm{g}$ of lysylendopeptidase at $37^{\circ} \mathrm{C}$ for $13 \mathrm{~h}$. The digested peptides were separated by HPLC on a $\mathrm{C}_{18}$ reverse phase column (Gilson) with a linear gradient between $0.05 \%$ trifluoroacetic acid (TFA) in water and $0.052 \%$ TFA in $80 \%$ acetonitrile (Ju et al., 2006). The aminoterminal amino acid sequences of the purified proteins and the internal peptides from HPLC were determined on an Applied Biosystem Procise automated gas-phase amino acid sequencer.

\section{Antibody, immunoblotting and immuno-precipitation experiment.} Polyclonal antibodies against rat FDH and SBTI were raised by injecting $20 \mu \mathrm{g}$ of the purified proteins into a male albino rabbit with complete Freund's adjuvant and giving a booster injection of the same amount of protein 14 days later. The resulting antibodies were affinity-purified as previously described (Cho et al., 1999). For immunoblotting, the proteins separated on the gel by electrophoresis were transferred electrophoretically to a polyvinylidene fluoride (PVDF) membrane and the membrane was immersed in $5 \%$ skim milk solution containing $1 \%$ horse serum for $12 \mathrm{~h}$, after which they were transferred to rinse solution I $(20 \mathrm{mM}$ Tris/ $\mathrm{HCl}, \mathrm{pH} 7.5$, containing $150 \mathrm{mM} \mathrm{NaCl}, 0.1 \%$ Tween 20 and $2.5 \%$ skim milk) containing affinity-purified antibody against FDH or SBTI protein $(50 \mathrm{ng} / \mathrm{ml})$ and kept at $4^{\circ} \mathrm{C}$ for $2 \mathrm{~h}$. The bound antibodies were identified using the ECL western blotting reagent kit (Amersham Life Science kit). Immuno-precipitation by antiFDH or anti-SBTI antibody was performed with $5 \mathrm{mg}$ proteins of rat liver homogenate, anti-FDH or anti-SBTI antibody and protein A-Sepharose $\left(100 \mu \mathrm{l}\right.$ of $50 \%$ suspended solution) at $4^{\circ} \mathrm{C}$ for $1 \mathrm{~h}$. The protein A-Sepharose beads were then washed extensively with washing buffer and then eluted with $2 \times$ SDS-PAGE loading buffer. The bound proteins eluted from the beads were separated on SDSPAGE and subjected to immunoblot.

Complex formation by SBTI injection in vivo. Male rats (200$300 \mathrm{~g})$ were injected in the tail vein with SBTI $(100 \mathrm{mg} / \mathrm{kg})$ in $100 \mu \mathrm{l}$ of saline. After $30 \mathrm{~min}$, the rat was killed by $\mathrm{CO}_{2}$ inhalation and then liver homogenate was obtained as described above. As a control, we have injected dimethylnitrosamine (DMN) with $50 \mathrm{mg} /$ $\mathrm{kg}$ body weight in $100 \mu \mathrm{l}$ of saline (Guo et al., 2006). The SBTI- or DMN-treated liver homogenates was separated on SDD-PAGE and subjected to immunoblot with anti-SBTI antibody.

Calibration curve. A calibration curve was prepared, following the instructions of the manufacturer of the column, by running blue dextran 2000, cytochrome $c(12 \mathrm{kDa})$, ovalbumin $(43 \mathrm{kDa})$, bovine serum albumin $(67 \mathrm{kDa})$, aldolase $(158 \mathrm{kDa})$, and catalase $(232$ $\mathrm{kDa})$, all from Amersham Biosciences. The elution of these markers was monitored by UV spectrophotometry $(280 \mathrm{~nm})$ with the full scale set to 0.05 absorbance units, and the elution volume was measured from the start of the sample application to the apex of the elution peak. Prior to the injection of a sample, a set of three markers (cytochrome $c$, ovalbumin, and catalase) was injected to test the performance of the column.

\section{(A)}

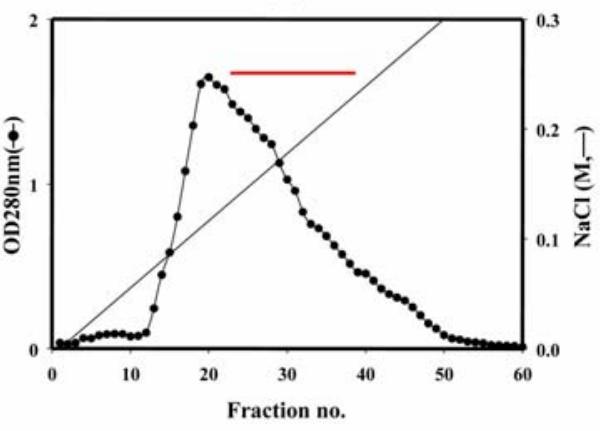

(C)

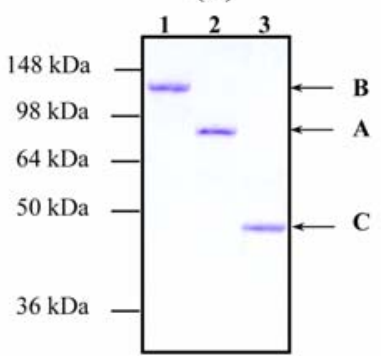

(B)

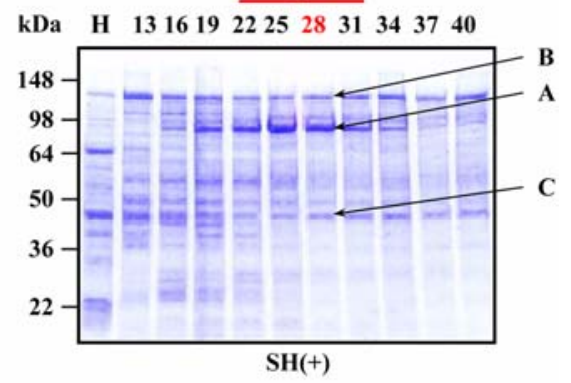

(D)

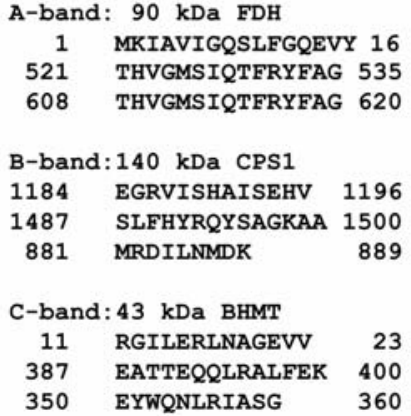

Fig. 2. Biochemical characterization of SBTI-binding proteins on SBTI-affinity column. (A), elution pattern of SBTI-affinity column. The fractions with red bars were collected and used for the next purification. (B), SDS-PAGE analysis pattern of (A). Lane H indicated the crude liver homogenate. The numbers on top of the gel indicate the fraction numbers of (A). (C), SDS-PAGE pattern of three SBTI-binding proteins after electro-elution. (D), the determined $\mathrm{N}$-terminal amino acid sequence of $90 \mathrm{kDa}$ protein and partial amino acid sequences of $140 \mathrm{kDa}, 90 \mathrm{kDa}$ and $43 \mathrm{kDa}$ proteins. 


\section{Results and Discussion}

Purification of SBTI-binding proteins from rat liver homogenate. To examine whether rat FDH can bind SBTI, we first carried out an SBTI-coupled Sepharose CL-4B column with rat liver homogenate. The bound proteins on the SBTI-coupled column were eluted with a 0-0.3 M NaCl linear gradient (Fig. 2A) and then each fraction was analyzed by SDS-PAGE under reducing conditions (Fig. 2B). A $90 \mathrm{kDa}$ protein band (band A) was enriched on several fractions (fraction No. 19 to 34 ). When we determined the amino terminal sequence of the $90 \mathrm{kDa}$ protein after transferring to PVDF membrane, the determined MKIAVIGQSLFGQEVY sequence was perfectly matched to the amino-terminal sequence of rat FDH (Fig. 2D), indicating that rat FDH protein can bind to SBTI. Interestingly, another two protein bands (bands B and C) with molecular masses of $140 \mathrm{kDa}$ and $43 \mathrm{kDa}$ were also co-eluted with rat FDH from the SBTIaffinity column. To characterize these two proteins, we have purified these two proteins to homogeneity by the electroelution method and then examined their purity by SDS-PAGE (Fig. 2C). When we determined the partial amino acid sequence of the $140 \mathrm{kDa}$ protein (band B), three partial amino acid sequences were perfectly matched to rat carbamoylphosphate synthetase 1 (CPS1, Fig. 2D). The $40 \mathrm{kDa}$ protein (band C) was identified as rat betaine homocysteine Smethyltransferase (BHMT, Fig. 2D). CPS1 is a rate-limiting enzyme catalyzing the first step of ureagenesis through the urea cycle in liver (Rubio and Cervera, 1995; Huo et al., 2005). This cycle is the body's main system for removing waste nitrogen produced by the metabolism of endogenous and exogenous protein. CPS1 is highly tissue specific, with function and production limited to the liver. BHMT is known to contribute to the regulation of homocysteine levels in plasma, which is deeply related with cardiovascular diseases (Pajares and Perez-Sala, 2006). Most plasma homocysteine is generated through the liver methionine cycle, in which BHMT metabolizes approximately $25 \%$ of this non-protein amino-acid. The liver is suggested as a main regulator of homocysteine levels (Jacobs et al., 2005). These results suggested that three liver enzymes, FDH, CPS1 and BHMT can be co-purified by an SBTI-coupled affinity column.

Rat FDH, CPS1 and BHMT make a complex on size exclusion column. To address a possibility that rat FDH, CPS1 and BHMT can make a protein complex in vitro, we further purified the FDH/CPS1/BHMT complex to homogeneity by gel-filtration columns. When the collected fractions containing FDH on the SBTI-affinity column (indicated by a red bar in Fig. 2A) were loaded onto a Sephacryl S-200 gel-
(A)

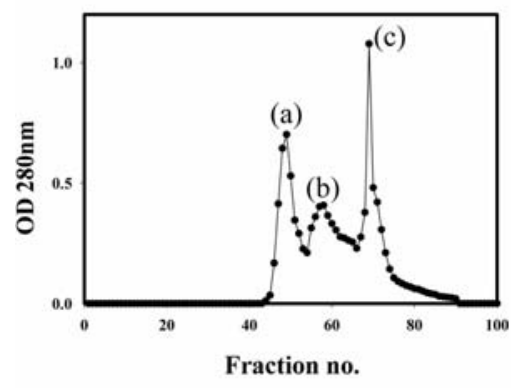

(C)

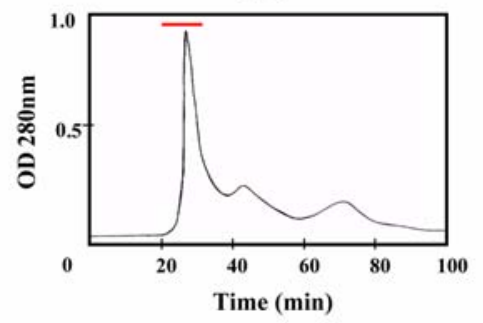

(B)

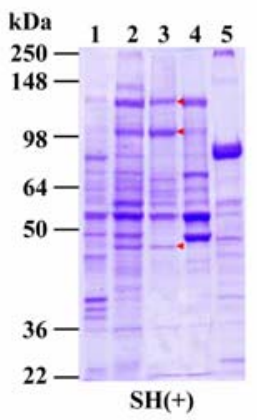

(D)

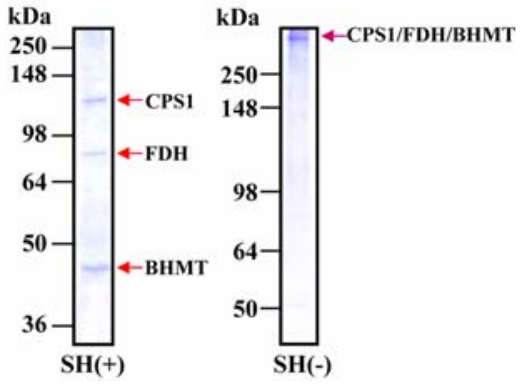

Fig. 3. The purification procedure of CPS1/FDH/BHMT complex by gel-filtration columns. (A), elution pattern of Sephacryl S-200 column. Each eluted peak is indicated as (a), (b) and (c). (B), SDS-PAGE pattern of (A). Lanes 1 and 6, the crude liver homogenate; lanes 2 and 7, the collected solution of SBTI-column (Fraction No. 22 $2^{\text {th }}-31^{\text {th }}$ ); lanes 3 and 8, 4 and 9, 5 and 10 indicated peak (a), (b) and (c) of Fig. 3A, respectively. (C), elution pattern of Superdex 200 FPLC column. (D), SDS-PAGE analysis patterns of the fractions indicated by red bar in Fig. 3C. 
(A)

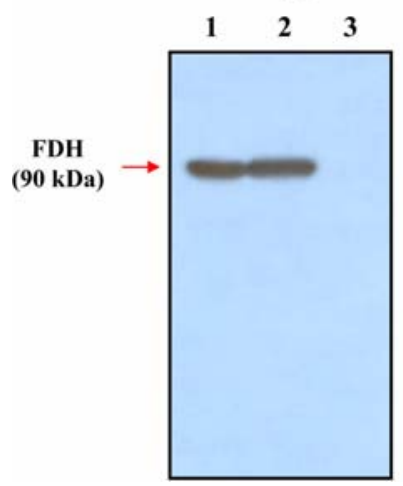

(B)

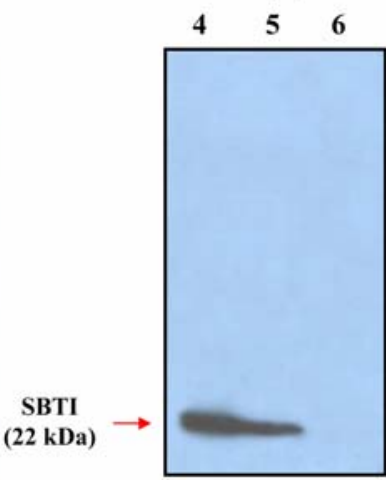

Fig. 4. The Western blot analysis after immuno-precipitation experiments. (A), The evidence of FDH/SBTI complex was obtained by using anti-FDH antibody. (B), anti-SBTI antibody was used for detection of FDH/SBTI complex. Lane 1, the purified FDH $(0.5 \mu \mathrm{g})$; lane 2, FDH/SBTI complex was detected with anti-FDH-antibody; lane 3, immuno-precipitation was carried out without anti-FDH antibody; lane 4, SBTI $(0.5 \mu \mathrm{g})$; lane 5, FDH/SBTI complex was detected with anti-SBTI antibody; lane 6, immuno-precipitation was carried out without anti-SBTI antibody.

filtration column, three peaks were generated (Fig. 3A) and SDS-PAGE analysis was carried out under reducing and nonreducing conditions (Fig. 3B). Among them, peak a contained three proteins as major protein components with molecular masses of $140 \mathrm{kDa}, 90 \mathrm{kDa}$ and $43 \mathrm{kDa}$ under reducing conditions (indicated by red arrows on lane 3 in Fig. 3B). However, the same peak showed as a major band at $\sim 300 \mathrm{kDa}$ molecular mass under non-reducing conditions (lane 8 in Fig. 3B), suggesting that FDH/CPS1/BHMT may make a complex in vitro. To further confirm this possibility, we loaded peak a of Sephacryl S-200 column onto a Superdex 200 FPLC gelfiltration column (Fig. 3C and 3D). As expected, the main peak showed three bands on reducing conditions (Fig. 3C) and one band with $\sim 300 \mathrm{kDa}$ molecular mass was identified (Fig. 3D). When each purified protein was independently applied to the same column, each CPS1, FDH, and BHMT corresponding to $140 \mathrm{kDa}, 90 \mathrm{kDa}$ and $43 \mathrm{kDa}$ peak, respectively, was observed (data not shown), indicating that each protein exists as a monomer in solution. Taken together, these results strongly demonstrated that CPS1/FDH/BHMT formed a protein complex in vitro.

\section{Complex Formation between SBTI and FDH was confirmed} by immuno-precipitation experiments. Immuno-precipitation analyses were carried out to ascertain that rat FDH can bind to SBTI in vitro. The rat liver homogenate, SBTI and anti-FDH or anti-SBTI antibody were mixed and then the generated complexes were precipitated by protein-A Sepharose resin. The precipitates were separated by SDS-PAGE under reducing conditions, and subsequently proteins were detected by Western blot analysis (Fig. 4). Rat FDH and SBTI complex

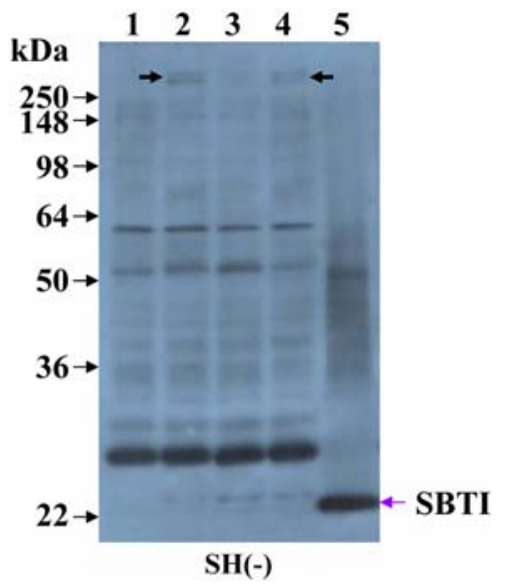

Fig. 5. CPS1/FDH/BHMT complex formation by SBTI injection in vivo. Lane 1, liver homogenate was obtained after saline injection; lane 2, liver proteins were obtained from SBTI-injected rat; lane 3, liver homogenate from $\mathrm{DMN}$-injected rat; lane 4, after DMN injection, 30 min later, SBTI was injected; lane 5, control SBTI $(0.5 \mu \mathrm{g})$ only.

was co-precipitated by anti-FDH (lane 2) or anti-SBTI antibody (lane 5), suggesting that FDH directly interacts with SBTI. On the other hand, the FDH-SBTI complex was not precipitated in the absence of antibodies (lanes 3 and 6). According to these observations, SBTI directly interacts with FDH and then this complex may recruit CPS1 and BHMT in liver homogenate.

Protein complex was also observed in liver homogenate after injection of SBTI in vivo. In order to examine whether the protein complex of FDH/CPS1/BHMT can be induced by SBTI injection in vivo, we injected SBTI into rat tail vein. After $30 \mathrm{~min}$, liver homogenate was obtained and analyzed by SDS-PAGE (12\%) under non-reducing conditions. The protein complex was identified with anti-SBTI antibody (Fig. 5). A $300 \mathrm{kDa}$ protein complex band was only detected in the liver homogenate after SBTI injection, but not after dimethylnitrosamine (DMN) injection as a control. This result ascertains our hypothesis that the Asp/His dyad catalytic site of liver FDH may have similar topology with the Asp/His catalytic site of serine proteases.

In this study, we found for the first time that SBTI can bind to rat liver FDH and that FDH/CPS1/BHMT molecules can make a complex in vitro and in vivo. We finally obtained $\sim 200$ $\mu \mathrm{g}$ of pure CPS1/FDH/BHMT complex from $1 \mathrm{~g}$ proteins of crude rat liver homogenate as a starting material, indicating that $\mathrm{CPS} 1 / \mathrm{FDH} / \mathrm{BHMT}$ proteins can be easily purified to homogeneity by using SBTI-affinity column. It was reported that the difficulties encountered in BHMT and CPS1 purification precluded detailed molecular studies for determining new biological functions (Pajares and Perez-Sala, 2006; Kothe et al., 2005). Therefore, the present demonstration will be useful information that has remained elusive in many previous 
studies regarding CPS1 and BHMT. Finally, our results provide that the topology between His/Asp catalytic residues of serine protease and His/Asp catalytic dyad residues of 10formyl-THF-utilizing enzymes may be similar in their enzyme activities.

Acknowledgment This work was supported for two years by Pusan National University Research Grant.

\section{References}

Bailey, L. B. and Gregory, J. F., 3rd (1999) Folate metabolism and requirements. J. Nutr. 129, 779-782.

Cho, M. Y., Lee, H. S., Lee, K. M., Homma, K., Natori, S. and Lee, B. L. (1999) Molecular cloning and functional properties of two early-stage encapsulation-relating proteins from the coleopteran insect, Tenebrio molitor larvae. Eur. J. Biochem. 262, 737-744.

Christeller, J. T. (2005) Evolutionary mechanisms acting on proteinase inhibitor variability. FEBS J. 272, 5710-5722.

Chumanevich, A. A., Krupenko, S. A. and Davies, C. (2004) The crystal structure of the hydrolase domain of 10formyltetrahydrofolate dehydrogenase: mechanism of hydrolysis and its interplay with the dehydrogenase domain. J. Biol. Chem. 279, 14355-14364.

Cook, R. J., Lloyd, R. S. and Wagner, C. (1991) Isolation and characterization of cDNA clones for rat liver 10formyltetrahydrofolate dehydrogenase. J. Biol. Chem. 266, 4965-4973.

Guo, L., Enzan, H., Hayashi, Y., Miyazaki, E., Jin, Y., Toi, M., Kuroda, N. and Hiroi, M. (2006) Increased iron deposition in rat liver fibrosis induced by a high-dose injection of dimethylnitrosamine. Exp. Mol. Pathol. 81, 255-261.

Huo, R., Zhu, H., Lu, L., Ying, L., Xu, M., Xu, Z., Li, J., Zhou, Z. and Sha, J. (2005) Molecular cloning, identification and characteristics of a novel isoform of carbamyl phosphate synthetase I in human testis. J. Biochem. Mol. Biol. 38, 28-33.

Inglese, J., Smith, J. M. and Benkovic, S. J. (1990) Active-site mapping and site-specific mutagenesis of glycinamide ribonucleotide transformylase from Escherichia coli. Biochemistry 29, 6678-6687.

Iwanaga, S., and Lee, B. L. (2005) Recent advances in the innate immunity of invertebrate animals. J. Biochem. Mol. Biol. 38, 128-150.
Jacobs, R. L., Stead, L. M., Devlin, C., Tabas, I., Brosnan, M. E., Brosnan, J. T. and Vance, D. E. (2005) Physiological regulation of phospholipid methylation alters plasma homocysteine in mice. J. Biol. Chem. 280, 28299-28305.

Ju, J. S., Cho, M. H., Brade, L., Kim, J. H., Park, J. W., Ha, N. C., Soderhall, I., Soderhall, K., Brade, H. and Lee, B. L. (2006) A novel $40-\mathrm{kDa}$ protein containing six repeats of an epidermal growth factor-like domain functions as a pattern recognition protein for lipopolysaccharide. J. Immunol. 177, 1838-1845.

Kothe, M., Purcarea, C., Guy, H. I., Evans, D. R. and Powers-Lee, S. G. (2005) Direct demonstration of carbamoyl phosphate formation on the C-terminal domain of carbamoyl phosphate synthetase. Protein Sci. 14, 37-44.

Krupenko, S. A., Wagner, C. and Cook, R. J. (1997) Domain structure of rat 10-formyltetrahydrofolate dehydrogenase. Resolution of the amino-terminal domain as 10-formyltetrahydrofolate hydrolase. J. Biol. Chem. 272, 10273-10278.

Lee, M. H., Osaki, T., Lee, J. Y., Baek, M. J., Zhang, R., Park, J. W., Kawabata, S., Soderhall, K. and Lee, B. L. (2004) Peptidoglycan recognition proteins involved in 1,3-beta-Dglucan-dependent prophenoloxidase activation system of insect. J. Biol. Chem. 279, 3218-3227.

Murthy, H. M., Clum, S. and Padmanabhan, R. (1999) Dengue virus NS3 serine protease. Crystal structure and insights into interaction of the active site with substrates by molecular modeling and structural analysis of mutational effects. J. Biol. Chem. 274, 5573-5580.

Odani, S., Odani, S., Ono, T. and Ikenaka, T. (1979) Proteinase inhibitors from a mimosoideae legume, Albizzia julibrissin. Homologues of soybean trypsin inhibitor (Kunitz). J. Biochem (Tokyo) 86, 1795-1805.

Pajares, M. A. and Perez-Sala, D. (2006) Betaine homocysteine Smethyltransferase: just a regulator of homocysteine metabolism? Cell Mol. Life Sci. 63, 2792-2803.

Perona, J. J. and Craik, C. S. (1995) Structural basis of substrate specificity in the serine proteases. Protein Sci. 4, 337-360.

Prezelj, A., Anderluh, P. S., Peternel, L. and Urleb, U. (2007) Recent advances in serine protease inhibitors as anticoagulant agents. Curr. Pharm. Des. 13, 287-312.

Rawlings, N. D., Tolle, D. P. and Barrett, A. J. (2004) Evolutionary families of peptidase inhibitors. Biochem. J. 378, 705-716.

Rubio, V. and Cervera, J. (1995) The carbamoyl-phosphate synthase family and carbamate kinase: structure-function studies. Biochem. Soc. Trans. 23, 879-883. 